\title{
The Structure and Physical Properties of Aramid Film Prepared by Electrodeposition of Poly(p-phenylene terephthalamide) Polyanion
}

\author{
Keiko KogA, Shigeyuki Ueta, and Motowo TAKAYANAGI \\ Faculty of Engineering, Kyushu Sangyo University, \\ Matsukadai, Fukuoka 813, Japan \\ (Received February 17, 1988)
}

\begin{abstract}
The electrodeposition of poly( $p$-phenylene terephthalamide) (PPTA) polyanion dissolved in dimethylsulfoxide (DMSO) formed a PPTA gel on the anode in a film state. By replacing DMSO in the gel with water and drying, a uniform PPTA film was obtained, which exhibited tensile strength of $155 \mathrm{MPa}$ and Young's modulus of $11 \mathrm{GPa}$. The structural variation from electrodeposited gel to annealed film was observed by the X-ray diffraction. The analysis revealed that the direction of the hydrogen bonded sheet of PPTA crystal in an unannealed film changed from perpendicular to parallel alignment with the film surface after strong annealing. The dynamic viscoelastic measurements of the film lead us to consider that the change of orientation of the hydrogen bonded sheet is brought about by rotational motion of molecular chains in crystal, corresponding to increased crystalline relaxation around $220^{\circ} \mathrm{C}$.

KEY WORDS Poly(p-phenylene terephthalamide) / Polyelectrolyte Electrodeposition / Hydrogen Bond / Dynamic Complex Modulus / Crystalline Relaxation /
\end{abstract}

$\operatorname{Poly}(p$-phenylene terephthalamide) (PPTA) is difficult to dissolve in solvents and is infusible. Only when a liquid crystalline dope of PPTA in sulfuric acid is spun, PPTA gives an ultrahigh modulus and ultrahigh strength fiber, Kevlar of du Pont Co. ${ }^{1}$ To prepare a film with the same method has been tried by several authors, ${ }^{2,3}$ using sulfuric acid solution with water coagulant.

We found a new preparation method of PPTA film, being quite different from the method of sulfuric acid solution and water coagulant, using the electrolysis of PPTA polyanion in organic solvent. One of the authors reported in a previous paper ${ }^{4}$ that the reagent formed by the reaction of sodium hydride and dimethylsulfoxide (DMSO) converted PPTA into polyanion with negative charge on a nitrogen atom in DMSO, resulting in a dark red homogeneous solution. The electrolysis of
PPTA polyanion formed a PPTA gel on the anode. Formation of PPTA gel was efficiently completed in a short time. By replacing DMSO in the gel with water and drying, a uniform PPTA film was obtained. The thickness of the film can be controlled by current and time at electrolysis.

This paper reports the preparation method of PPTA film by electrodeposition, the structural change from the electrodeposited PPTA gel to the annealed film and the mechanical properties of the film. We discuss the mechanism of observed crystalline relaxation in connection with orientation of hydrogen bonded sheet of crystal.

\section{EXPERIMENTAL}

\section{Preparation of Electrolytic Solution} PPTA of $\bar{M}_{v}=50000$ was supplied by Asahi 
Chemical Industry Co., Ltd. in powder form. The metalation reaction of PPTA was accomplished by the procedure of Takayanagi and Katayose. $^{4} 0.02 \mathrm{~mol}(0.84 \mathrm{~g})$ of sodium hydride and 11 of DMSO were placed in a three necked separable flask equipped with a nitrogen inlet, drying tube, thermometer and stirrer. The mixture was heated to $70^{\circ} \mathrm{C}$ for a period of
$20 \mathrm{~min}$ and maintained at $70^{\circ} \mathrm{C}$ for $40 \mathrm{~min}$ under nitrogen with stirring. The reacted ingredient was cooled to room temperature, and to which $0.02 \mathrm{~mol}$ amide unit $(2.5 \mathrm{~g})$ of PPTA was added. Stirring the mixture at $30^{\circ} \mathrm{C}$ for a period of $48 \mathrm{~h}$, PPTA was dissolved in DMSO to form polyanion of PPTA. The reaction scheme is represented by

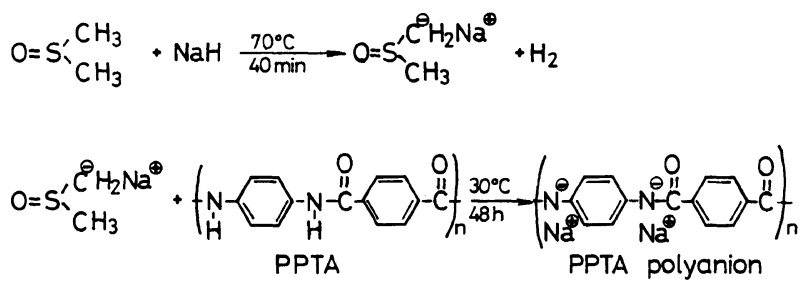

I

\section{Electrodeposition of PPTA Polyanion}

Figure 1 shows the apparatus for electrolysis of PPTA polyanion. A solution of I in DMSO filled the space between coaxial double cylinders, the outer cylinder being cathode and the inner cylinder being anode. Both cylinders were made of stainless steel. The outer cylinder was fixed and the inner cylinder was rotated at a constant speed of $280 \mathrm{rpm}$. The electrolysis was conducted galvanostatically. Current density was $1-3 \mathrm{~mA} \mathrm{~cm}^{-2}$ and the electrolysis was continued for 5-90 $\mathrm{min}$.

Just after electrodeposition, the film was a gel. This gel was taken off from the electrode.

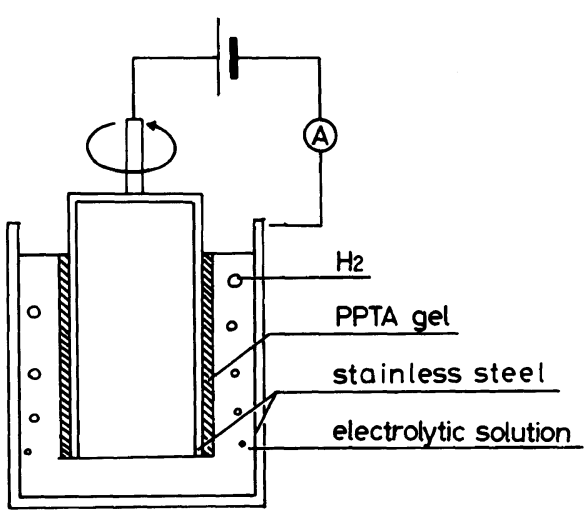

Figure 1. Apparatus for electrolysis of PPTA polyanion.
After replacing DMSO in the gel with $1 N$ aqueous hydrochloric acid and simultaneously neutralizing $\mathrm{Na}^{+}$ions contained in the gel, the gel was washed with water and dried at room temperature for one day.

The thickness of the film ranging from 3$25 \mu \mathrm{m}$ was proportional to the charge density of electrolysis. Films of $3-16 \mu \mathrm{m}$ thickness were used. Annealing of the films was carried

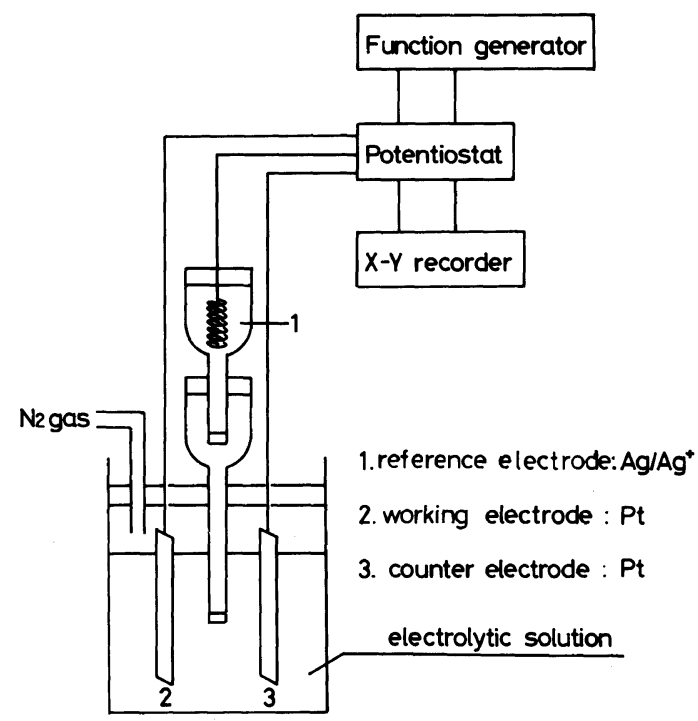

Figure 2. Apparatus for measurement of electrolysis curve. 
out in a nitrogen atmosphere.

\section{Measurement}

Electrolysis curve (E-I curve) was measured using a potentiostat (Hokuto Electric) shown in Figure 2. Pt plates were used as both working and counter electrodes, and an $\mathrm{Ag} / \mathrm{Ag}^{+}$electrode was used for reference. Differential scanning calorimetry (DSC) was performed on a Rigaku Denki DSC-8230 at a heating rate of $10^{\circ} \mathrm{C} / \mathrm{min}$ under nitrogen gas flow. Wide angle $\mathrm{X}$-ray photographs were taken by the Ni-filtered $\mathrm{Cu} K_{\alpha}$ radiation. The density was measured by the floating method in a carbon tetrachloride-ethanol mixture at $25^{\circ} \mathrm{C}$. Mechanical properties were measured by a Tensilon tensile tester and Rheovibron DDV-III viscoelastometer (Orientec).

\section{RESULTS AND DISCUSSION}

\section{Electrolysis Curves}

Figure 3 shows the electrolysis curves of (a) PPTA polyanion and (b) methylsulfinylcarbanion in DMSO at a potential sweep rate of $50 \mathrm{mVs}^{-1}$. The oxidation potential of the PPTA polyanion is lower than that of methylsulfinylcarbanion. The electrolytic current of PPTA polyanion saturates at higher potential, while for methylsulfinylcarbanion, the current increases linearly with increasing potential. This saturation results from decrease in the

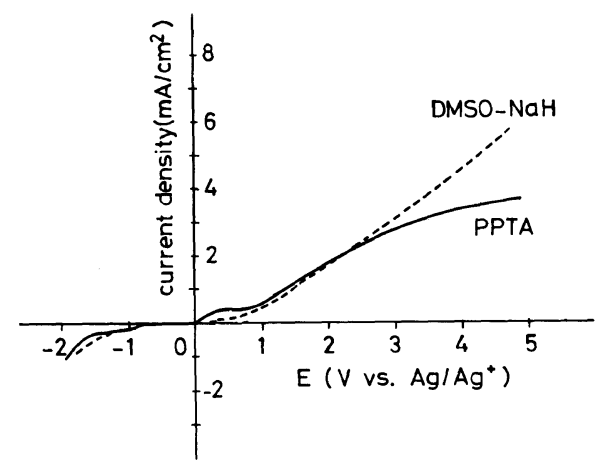

Figure 3. Electrolysis curves for (a) PPTA polyanion and (b) methylsulfinylcarbanion in DMSO. rate of diffusion of polyanion by deposited PPTA.

Electrodeposition of PPTA polyanion is very efficient. This might be explained by the fact that the PPTA polyanion is rodlike due to the resonance structure along the whole molecular chain ${ }^{5}$ and the discharge process of electron takes place along the whole chain length. In fact, the electrolysis of the aramid copolymer polyanion (II), poly( $p$-phenyleneco-3, 4'-oxydiphenylene terephthalamide) polyanion, which contains molecular coil segments, cannot deposit a uniform gel on the anode.

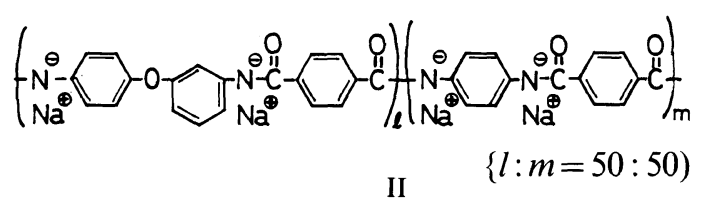

The mechanisms for electrolytic reaction at electrodes are presently assumed to be as follows.

(Cathode)

$$
\begin{aligned}
& \mathrm{Na}^{+}+\mathrm{e} \longrightarrow \mathrm{Na} \\
& \mathrm{Na}+\mathrm{DMSO} \longrightarrow \mathrm{O}=\mathrm{S}_{-}^{-}-\mathrm{CH}_{3} \mathrm{CH}_{2}+\frac{1}{2} \mathrm{H}_{2}
\end{aligned}
$$

(Anode)

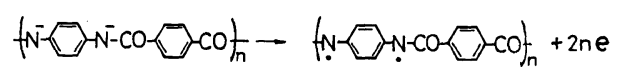

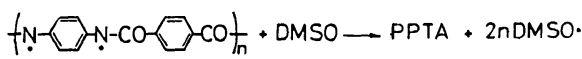

\section{Structural Change of Electrodeposited PPTA Film}

The PPTA gel film as electrodeposited was transparent and 15 times the weight of the dried film. After being soaked in $1 N$ aqueous hydrochloric acid and washed with water, the gel became opaque. DMSO in the gel was replaced by water and the volume of the gel decreased to about $2 / 3$ the original one. Figure 4 shows X-ray diffraction photographs of (a) the gel containing DMSO and (b) the gel containing water. In Figure 4, the direction of 


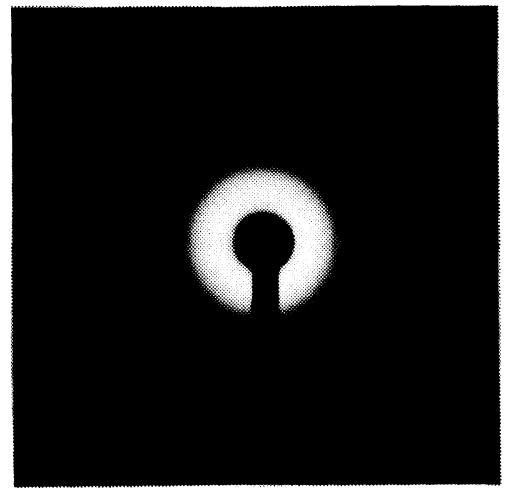

(a) PPTA gel/DMSO

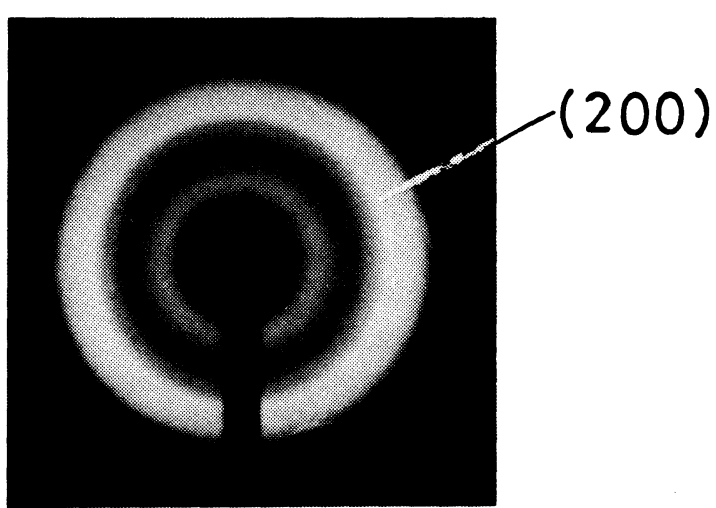

(b) PPTA gel/ $\mathrm{H}_{2} \mathrm{O}$

Figure 4. Wide angle X-ray diffraction photographs taken from the edges of electrodeposited PPTA gel film containing (a) DMSO and (b) $\mathrm{H}_{2} \mathrm{O}$.

incident X-ray beam is parallel to the gel surface. The diffraction patterns taken by the incident beam normal to the gel surfaces of both containing DMSO and water are the same as in Figure 4. For the gel containing DMSO, no diffraction due to PPTA crystals was observed but a strong hallo due to DMSO was seen. For the gel containing water, the diffraction rings due to PPTA crystals were clearly observed.

The crystal structure of PPTA reported by Northolt ${ }^{6}$ and Tadokoro et al. ${ }^{7}$ is monoclinic with $a=0.780 \mathrm{~nm}, b=0.519 \mathrm{~nm}, c=1.29 \mathrm{~nm}$ and $\gamma=90^{\circ}$ and contains two polymer chains. This crystal structure is designated as form I. The sharp ring in Figure $4(\mathrm{~b})$ is the (200) reflection of form I. The (004) reflection $(d=0.323 \mathrm{~nm})$ is broad and the $(110)$ reflection is absent. This diffraction pattern agrees with that of swollen film reported in the previous paper, ${ }^{8}$ which was prepared by coagulating sulfuric acid solution with water and not dried. According to the author of this paper, PPTA molecules align regularly in the direction of $a$ axis in which PPTA molecules are combined by van der Waals force, while the arrangement of PPTA molecules is disordered along the $b$ axis, in a hydrogen bond direction, because hydrogen bond formation among the PPTA molecules is disturbed by water molecules. This state is called the "liquid crystal-like swollen structure". 8 Once dried to a film, it never returns to the swollen state as before.

PPTA film is obtained by drying the gel film containing water at room temperature. Figure 5 shows the X-ray diffraction photographs of unannealed PPTA film (a) and the PPTA film annealed at $450^{\circ} \mathrm{C}$ for $10 \mathrm{~s}$ (b). In this figure, the patterns taken by incident X-ray beam normal and parallel to the film surface are designated through view (TV) and the edge view (EV), respectively. Figure 6 shows a schematic representation of the edge views in Figure 5. In the TV photograph of unannealed PPTA film (a), Debye rings are all assigned based on form I and additionally, the (200) reflection is more intense than the (110) reflection. In the EV photograph, the (110) reflection is intensified on the equator and the (200) and (006) reflections are intensified on the meridian. The ac-plane of the crystal is thus parallel to the film surface; that is, the hydrogen bonded sheet is perpendicular to the film surface. This crystal orientation is shown schematically in Figure 7(a). Haraguchi et $a l^{2,8}$ reported that in PPTA film obtained by coagulation of sulfuric acid solution with water, the crystal modification called form II was formed. The cell of form II $(a=0.80 \mathrm{~nm}$, $b=0.51 \mathrm{~nm}, c=1.29 \mathrm{~nm}$ ) is similar to form $\mathrm{I}$, 


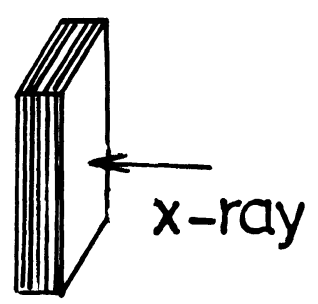

\section{through}
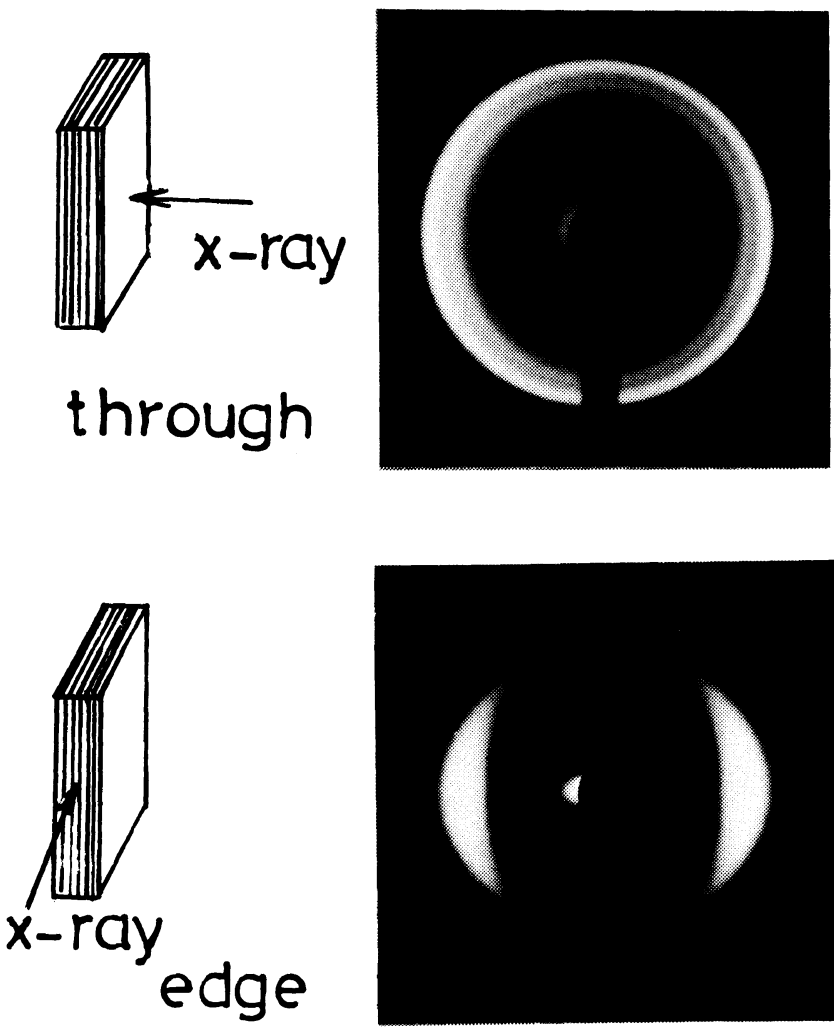

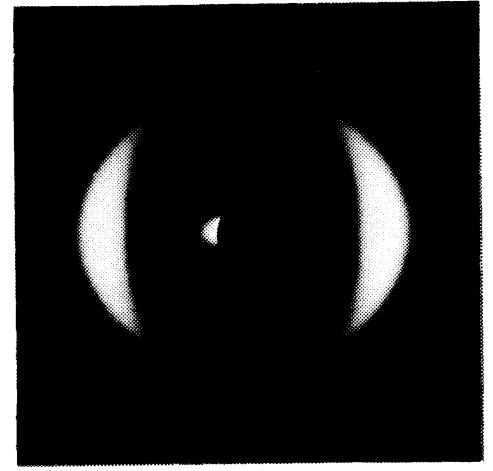

(a) Unannealed
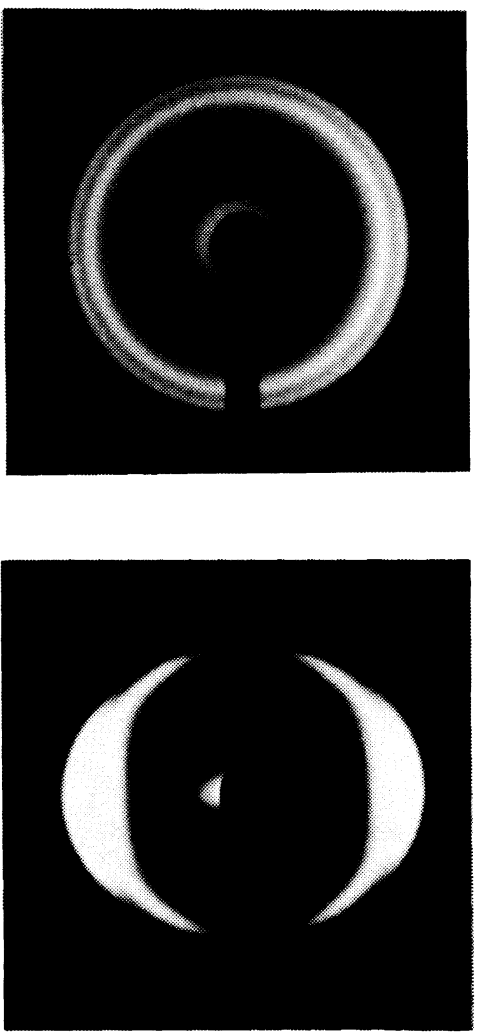

(b) Annealed

Figure 5. X-Ray diffraction photographs of PPTA films (a) unannealed and (b) annealed at $450^{\circ} \mathrm{C}$.

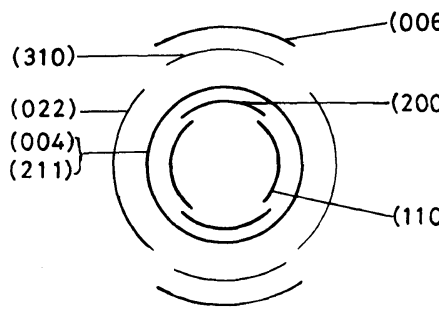

(a) Unannealed

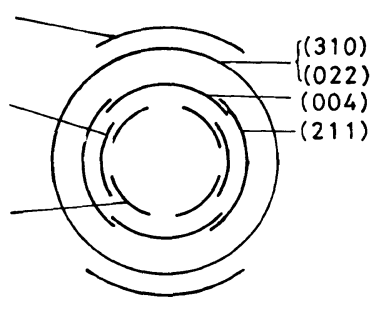

(b) Annealed

Figure 6. Schematic diagrams of observed X-ray photographs taken from the edge for (a) unannealed and (b) annealed PPTA films shown in Figure 5.

but the packing of molecules is different from form $I$ as shown in Figure $8 .^{8}$ In the electrodeposited PPTA film, form II crystal was not identified, judging from the absence of the (010) reflection characteristic of form II which appears at $2 \theta \cong 17^{\circ}$ as inner peak. However, in both films obtained by electrodeposition by us and coagulation of sulfuric acid solution by Haraguchi et al., ${ }^{2}$ the hydrogen bonded sheet of the crystals is perpendicular to the film surface.

For the annealed PPTA film, the intensities of the (200) and (110) reflections in the TV photograph are reversed and in the EV photo- 


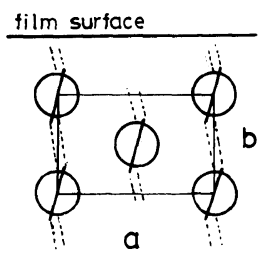

(a) Unannealed

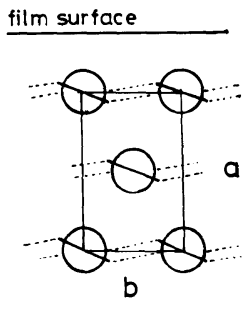

(b) Annealed
Figure 7. Schematic representation of crystal orientation in PPTA films.

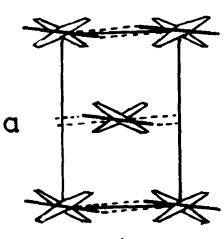

$b$

Form I

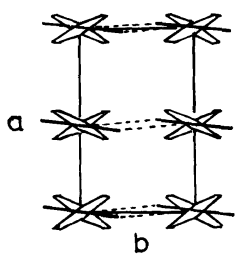

Form II
Figure 8. $c$ axis projections of the crystal structure for (a) form $\mathrm{I}^{6}$ and (b) form II. ${ }^{8}$

graph, the (200) reflection is intensified on the equator and the (110) reflection splits into four spots. It is thus apparent that the hydrogen bonded sheet changes to the parallel alignment to the film surface by annealing (see Figure 7(b)). This orientational change by annealing accords with the results of the PPTA film obtained by coagulation of sulfuric acid solution with water. ${ }^{2}$ This orientational change begins at about $300^{\circ} \mathrm{C}$. We will discuss hereafter the origin of the change of orientation referring to mechanical relaxation data.

Figure 9 shows the DSC curves of electrodeposited PPTA film. A large endothermic peak around at $80^{\circ} \mathrm{C}$ appears at the first run of heating curve of unannealed PPTA film, being reduced largely at the second run of the heating process after heating to $400^{\circ} \mathrm{C}$ in the first run. The extent of decrease at the peak varies from sample to sample.

We first considered that this peak was due to removal of water molecules incorporated into PPTA crystals, as $\mathrm{Xu}$ et al. $^{\left.{ }^{9}\right)}$ reported that PPTA formed a crystalline solvate containing

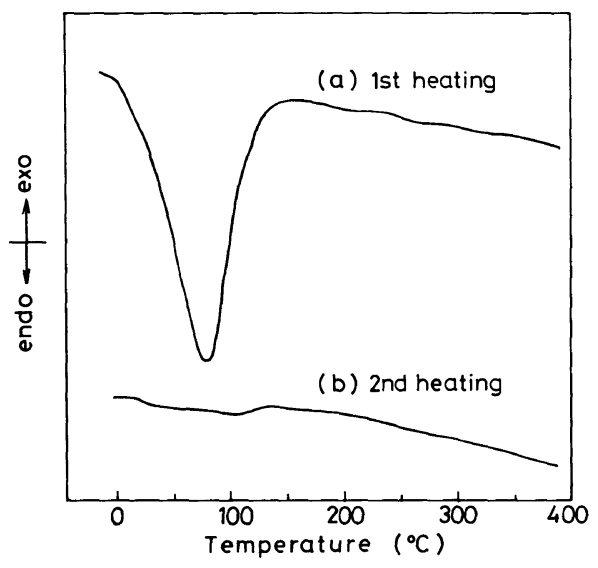

Figure 9. DSC curves of PPTA film.

sulfuric acid molecules or water molecules in its crystals. As can be seen in Figure 5(a), however, there exist no reflections to suggest crystalline solvate (hydrate). Also no expansion of intermolecular distance was observed. Therefore the water molecules associated with the large endothermic peak of DSC curve are possibly combined by hydrogen bonds to an amide group of PPTA in a disordered region or fibrillar surface with crystalline texture.

The structural changes mentioned above are summarized in Figure 10.

Figure 11 shows a SEM photograph of the fractured surface of unannealed PPTA film. Many layers parallel to the film surfac are observed in the fractured surface. In practice, it frequently occurred that another layer of gel was torn off from the electrodeposited gel.

\section{Mechanical Properties}

Table I lists the results of tensile testing, the absolute values of the tensile complex moduli observed at $110 \mathrm{~Hz}$ and the densities for the PPTA films. A slight anisotropy in modulus was found by the rotating electrodes. The tensile modulus and tensile strength of the electrodeposited film are comparable to those of the film obtained from sulfuric acid solution. By annealing, the modulus increased but the strength decreased. The increase of the 
Aramid Film

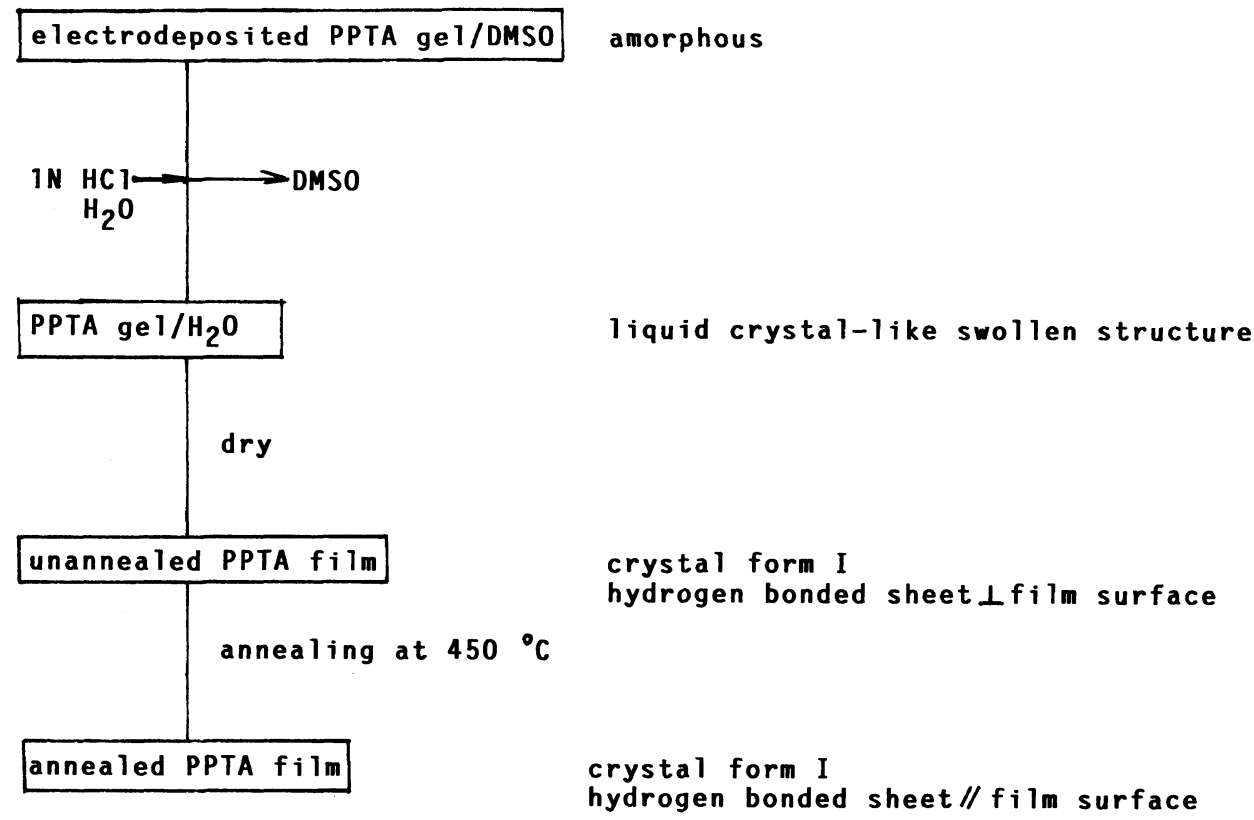

Figure 10. Block diagram representing structure change from electrodeposited PPTA gel to annealed film.

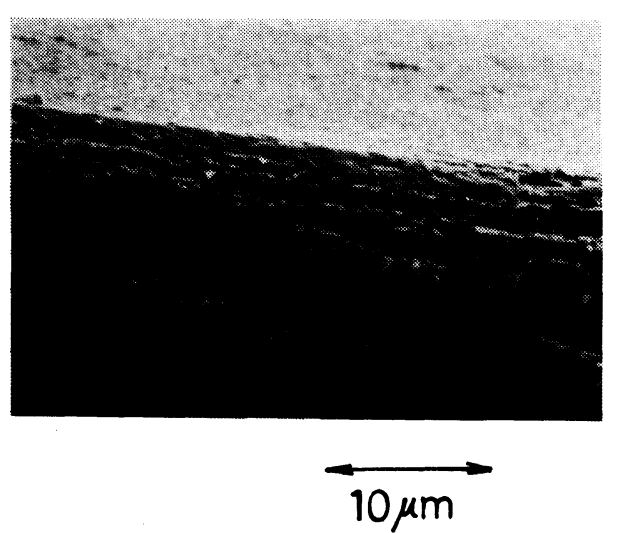

Figure 11. SEM photograph of fractured section of PPTA film.

modulus is attributed to enhanced crystallinity as indicated by the increase of density.

Figure 12 shows the temperature dependence of the tensile storage moduli $\left(E^{\prime}\right)$ and loss tangent $(\tan \delta)$ measured at $110 \mathrm{~Hz}$ for the unannealed and annealed PPTA films. Although $E^{\prime}$ decreases gradually with temperature for unannealed film, the value of 7

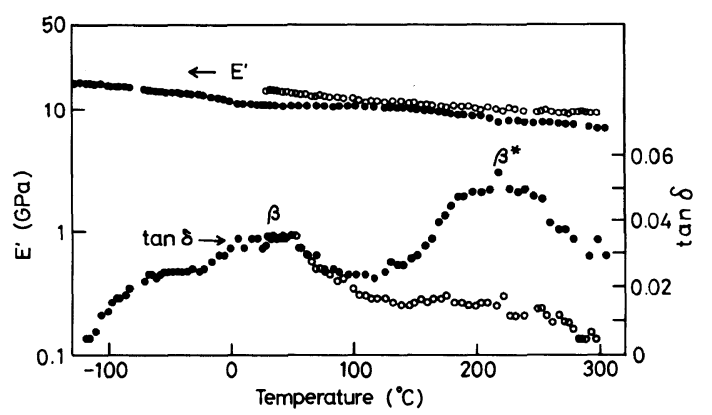

Figure 12. Temperature dependence of $E^{\prime}$ and $\tan \delta$ at $110 \mathrm{~Hz}$ for unannealed (filled circles) and annealed (open circles) PPTA films.

$\mathrm{GPa}$ is maintained at $300^{\circ} \mathrm{C}$, indicating excellent heat resistance. Two broad peaks around $30^{\circ} \mathrm{C}$ and $220^{\circ} \mathrm{C}$ are observed in the $\tan \delta$ curve of the unannealed film, which agrees with the results of Kevlar fiber in the previous paper, ${ }^{10}$ but the peak heights observed here in a film form are larger than those they observed. For annealed film, it is diffcult to measure the modulus at low tempertures because of its fragility. By annealing, $E^{\prime}$ increased and the 
Table I. Mechanical properties and densities of electrodeposited PPTA films

\begin{tabular}{|c|c|c|c|c|c|c|}
\hline \multirow{2}{*}{ Sample } & & $\begin{array}{l}\text { Tensile } \\
\text { modulus }\end{array}$ & $\begin{array}{l}\text { Tensile } \\
\text { strength }\end{array}$ & Extensibility & $|E|^{\mathrm{a}}$ & Density \\
\hline & & $\mathrm{GPa}$ & $\mathrm{MPa}$ & $\%$ & $\mathrm{GPa}$ & $\mathrm{g} \mathrm{cm}^{-3}$ \\
\hline \multirow[t]{2}{*}{ Unannealed } & $/ / \mathrm{b}^{\mathrm{b}}$ & 7.0 & 155 & 6.9 & 10.8 & 1.356 \\
\hline & $\perp^{\mathrm{c}}$ & 4.6 & 101 & 6.7 & 8.9 & \\
\hline \multirow[t]{2}{*}{ Annealed $^{d}$} & $/ /$ & 7.3 & 103 & 2.1 & 14.1 & 1.427 \\
\hline & $\perp$ & 5.1 & 70 & 1.8 & - & \\
\hline
\end{tabular}

a $110 \mathrm{~Hz}$.

b Paralell to the rotating direction.

c Perpendicular to the rotating direction.

d $400^{\circ} \mathrm{C}$.

$\tan \delta$ peak around $220^{\circ} \mathrm{C}$ decreased remarkably. The relaxation around $30^{\circ} \mathrm{C}$ is designated as the $\beta$ relaxation and the one around $220^{\circ} \mathrm{C}$ as the $\beta^{*}$ relaxation. The $\beta$ relaxation is associated with the disordered structure being accessible to water molecules. The $\beta^{*}$ relaxation might be associated with the inside of the crystals.

Now we discuss the mechanism of the $\beta^{*}$ relaxation. As mentioned above, for the unannealed film, the hydrogen bonded sheet of crystal is perpendicular to the film surface. This orientation resulted from preferential aggregation of the PPTA molecules in the direction of van der Waals bonding (in the direction of $a$-axis) and parallel alignment of $a c$-plane to the film surface when the PPTA molecules crystallized from the gel swollen with water. ${ }^{10}$ The orientation of hydrogen bonded sheet changed to parallel alignment to the film surface by annealing above $300^{\circ} \mathrm{C}$ (see Figure 7). This parallel alignment of the hydrogen bonded sheet is designated as state 1 and the perpendicular one as state 2. State 2 is less stable and at a higher energy level than state 1 because hydrogen bonds are in an unbound state on the layer surface. The layers of PPTA with thickness of several hundreds $\mathrm{nm}$ are stacked to form an electrodeposited PPTA film, the structure of which is clearly seen in Figure 11. In order to change from state 2 to state 1 , the molecular chains must rotate about their axes by an angle of $90^{\circ}$ and their locations must change slightly. Such molecular motions should be observed as mechanical relaxation related to crystal. The $\beta^{*}$ relaxation observed here corresponds to this. Actually, Haraguchi et al. ${ }^{10}$ assigned the relaxation around $220^{\circ} \mathrm{C}$ to the crystalline relaxation based on increased relaxation magnitude with increasing density and independence on humidity.

Panar et al. ${ }^{11}$ estimated the long period of Kevlar 49 fiber as $35 \mathrm{~nm}$ and showed by etching and the EM method that this length was the period of defect bands. Assuming the same value for the long period of the film, 27 monomer units are included in this period. It is considered that the activation energy of the $\beta^{*}$ relaxation corresponds to the energy barrier associated with the molecular chain of the long period length. On the other hand, the energy difference between states 1 and 2 corresponds to the energy to form hydrogen bonds of unbound molecules included in the same chain length. The number of unbound NH linkages or CO linkages associated with this variation at the crystal surface is 54 . Using the value of hydrogen bond energy of $\mathrm{N}-\mathrm{H}---\mathrm{O}$ in acetamide $\left(\mathrm{CH}_{3} \mathrm{CONH}_{2}\right){ }^{12} 14.6 \mathrm{~kJ} \mathrm{~mol}^{-1}$, the amount of energy difference between states 1 and 2 is given as $788 \mathrm{~kJ} \mathrm{~mol}^{-1}$. Using the activation energy of $209 \mathrm{~kJ} \mathrm{~mol}^{-1}$ reported by Haraguchi et al. ${ }^{10}$ for the $\beta^{*}$ relaxation, the 


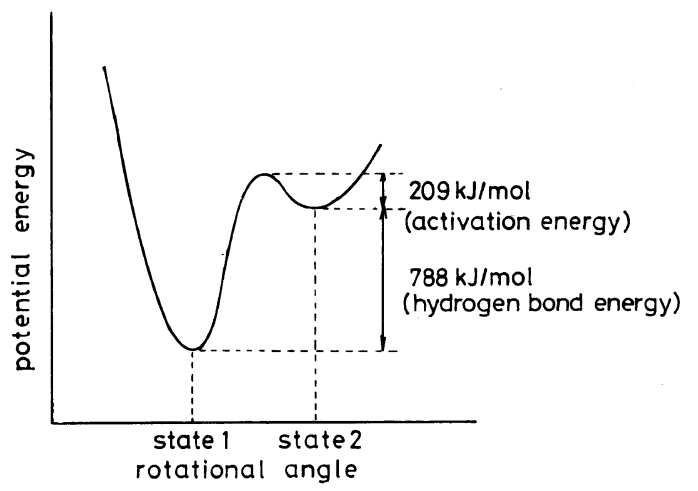

Figure 13. Potential energy curve for state 1: hydrogen-bonded sheet // film surface and for state 2: hydrogen-bonded sheet $\perp$ film surface.

energy curve is schematically represented in Figure 13. Once the transition from state 2 to state 1 takes place by annealing in a crystal and the viscoelastic relaxation is completed, the crystal cannot return to state 2 .

\section{CONCLUSIONS}

Poly( $p$-phenylene terephthalamide) (PPTA) film was prepared by electrodeposition of PPTA polyanion dissolved in dimethylsulfoxide (DMSO). A gel state of PPTA was formed on the anode. A uniform film obtained by washing the gel and drying has a Young's modulus of $11 \mathrm{GPa}$ and tensile strength of 155 MPa.

PPTA is amorphous in the gel as electrodeposited. Replacing DMSO in the gel with water, PPTA becomes a liquid crystal-like swollen structure. The film obtained by drying this is the aggregation of crystallites in which hydrogen-bonded sheets align perpendicular to the film surface. The orientation of hydrogen-bonded sheets changed to parallel alignment to the film surface by annealing through rotation of molecules around the molecular chain axes; this is related to the mechanical relaxation around $220^{\circ} \mathrm{C}$.

\section{REFERENCES}

1. S. L. Kwolek, U. S. Patent., 3,671,541 (1972).

2. K. Haraguchi, T. Kajiyama, and M. Takayanagi, $J$. Appl. Polym. Sci., 23, 903 (1979).

3. H. Bodaghi, T. Kitao, J. E. Flood, J. F. Fellers, and J. L. White, Polym. Eng. Sci., 24, 242 (1984).

4. M. Takayanagi and T. Katayose, J. Polym. Sci., Polym. Chem. Ed., 19, 1133 (1981).

5. K. Tashiro, M. Kobayashi, and H. Tadokoro, Macromolecules, 10, 413 (1977).

6. M. G. Northolt, Eur. Polym. J., 10, 799 (1974).

7. R. Hasegawa, Y. Chatani, and H. Tadokoro, Abstracts, Meeting of Society of Crystallography Japan, Osaka, Japan, 1973, p 21.

8. K. Haraguchi, T. Kajiyama, and M. Takayanagi, $J$. Appl. Polym. Sci., 23, 915 (1979).

9. D. Xu, K. Okuyama, F. Kumamaru, and M. Takayanagi, Polym. J., 16, 31 (1984).

10. K. Haraguchi, T. Kajiyama, and M. Takayanagi, Sen-i Gakkaishi, 23, T-535 (1977).

11. M. Panar, P. Avakian, R. C. Blume, K. H. Gardner, T. D. Gierke, and H. H. Yang, J. Polym. Sci., Polym. Phys. Ed., 21, 1955 (1983).

12. M. Haisa, "Gendai Butsurigaku Koza 13," Tokyo Kagaku Dojin, Tokyo, 1967. 